\title{
Dynamic simulations of transcriptional control during cell reprogramming reveal spatial chromatin caging
}

Marco Di Stefano ${ }^{1,2, *}$, Ralph Stadhouders ${ }^{2, \#}$, Irene Farabella ${ }^{1,2}$, David Castillo ${ }^{1,2}$, François Serra $^{1,2, \wedge}$, Thomas Graf ${ }^{2, *}$, and Marc A. Marti-Renom ${ }^{1,2,3,4, *}$

1. CNAG-CRG, Centre for Genomic Regulation (CRG), Barcelona Institute of Science and Technology (BIST), Baldiri i Reixac 4, 08028 Barcelona, Spain.

2. Centre for Genomic Regulation (CRG), Barcelona Institute of Science and Technology (BIST), Dr. Aiguader 88, 08003 Barcelona, Spain.

3. Universitat Pompeu Fabra (UPF), 08002 Barcelona, Spain.

4. ICREA, Pg. Lluís Companys 23, 08010 Barcelona, Spain

${ }^{\wedge}$ Current address: Computational Biology Group - Barcelona Supercomputing Center (BSC), 08034 Barcelona, Spain

\#Current address: Department of Pulmonary Medicine and Department of Cell Biology, Erasmus MC, Rotterdam, the Netherlands

*To whom correspondence should be addressed: M.A.M-R. martirenom@cnag.crg.eu, M.D.S. marco.distefano@cnag.crg.eu and T.G, thomas.graf@crg.eu 


\section{ABSTRACT}

Chromosome structure is a crucial regulatory factor for a wide range of nuclear processes. Chromosome Conformation Capture (3C)-based experiments combined with computational modelling are pivotal for unveiling 3D chromosome structure. Here, we introduce TADdyn, a new tool that integrates time-course $3 \mathrm{C}$ data, restraint-based modelling, and molecular dynamics to simulate the structural rearrangements of genomic loci in a completely data-driven way. We applied TADdyn on in-situ Hi-C time-course experiments studying the reprogramming of murine $B$ cells to pluripotent cells, and characterized the structural rearrangements that take place upon changes in the transcriptional state of 11 genomic loci. TADdyn simulations show that structural cages form around the transcription starting site of active loci to stabilize their dynamics, by initiating (hit) and maintaining (stick) interactions with regulatory regions. Consistent findings with TADdyn for all loci under study suggest that this hit-and-stick mechanism may represent a general mechanism to trigger and stabilize transcription. 


\section{INTRODUCTION}

The three-dimensional (3D) structure of the genome has been shown to modulate transcriptional regulation ${ }^{1-3}$ and to play a role in cancer and developmental abnormalities ${ }^{4}$. In the effort of characterising 3D genome structures, Chromosome Conformation Capture (3C)-based experiments ${ }^{5}$ allow to capture a single snapshot of the genome conformation at a given time. A plethora of theoretical approaches have been developed to take advantage of 3C-based experimental data and model genome spatial organization. Restraint-based modelling approaches ${ }^{6}$ take $3 \mathrm{C}$-based contact frequencies as input and employ ad hoc conversions to spatial distances for determining 3D genome structure ${ }^{7-12}$. This approach has provided valuable insights into the structural organization of chromosomal regions in various organisms ${ }^{13}$. Complementary, thermodynamics-based approaches ${ }^{14-22}$ use physics-based principles to test specific interactions or interaction mechanisms to explain the molecular origins of the contact patterns obtained in 3C-based experiments. Together, these theoretical approaches provide insights into chromatin conformation ${ }^{16,17,23,24}$ and the possible mechanisms that form chromosome territories ${ }^{18}$, compartments ${ }^{19}$ and Topologically Associating Domains (TADs) 20,22,25,26.

Decreased sequencing costs together with more refined experimental protocols has recently permitted performing 3C-based time-resolved experiments to monitor genome conformation dynamics of biological processes at high resolution. For example, Hi-C experiments have been applied to study nuclear organization during mitosis $^{27,28}$ or meiosis ${ }^{29-31}$, perturbations induced by hormone treatment ${ }^{32}$ and during induced neural differentiation ${ }^{33}$ or cell reprogramming ${ }^{34}$. However, none of the strategies mentioned above can currently take full advantage of these new datasets, and computational approaches specifically designed for the simulation of timedependent conformational changes (4D) are not yet available. To fill this gap, we introduce TADdyn, a novel computational method which allows modeling 3D structural transitions of chromatin using time-resolved $\mathrm{Hi}-\mathrm{C}$ datasets combining restraint-based modelling and molecular dynamics. We found two distinct phases of 3D genome conformation dynamics by applying TADdyn to 11 representative gene loci during cell reprogramming of mouse pre-B lymphocytes into Pluripotent Stem 
Cells (PSCs) ${ }^{34}$. First, the transcription start site (TSS) forms a 'cage'-like structure within a TAD, favoring specific contacts with open and active (enhancer) regions that may be located several kilo-bases (kb) away (here called the 'hit phase'). Second, the cage confines and stabilizes the dynamics of the TSS and its interactions with active regions against simulated thermal motion (the 'stick phase'). We propose this hit-and-stick model as a general mechanism for chromatin architectural changes linked to transcriptional activation. 


\section{RESULTS}

The TADdyn modelling strategy. TADdyn is based on the following methodological steps (Methods and Fig. 1): (i) collection of experimental data, (ii) representation of selected chromatin regions using a bead-spring polymer model, (iii) conversion of experimental data into time-dependent restraints, (iv) application of steered molecular dynamics to simulate the adaptation of chromatin models to satisfy the imposed restraints, and (v) analysis of the spatial conformations obtained.

We applied TADdyn to a previously published in-situ Hi-C interaction time-series dataset (GEO accession number GSE96611). This included seven time-points of insitu $\mathrm{Hi}-\mathrm{C}$ experiments during $\mathrm{C} / \mathrm{EBP} \alpha$ priming followed by Oct4, Sox2, Klf4 and Myc (OSKM)-induced reprogramming of $\mathrm{B}$ cells to $\mathrm{PSCs}^{34}$. We focused on 10 different $\sim 2 \mathrm{Mb}$ regions of the mouse genome encompassing the promoters of 11 genes of interest. We selected these specific genes because they are representative of different time-dependent patterns of transcriptional activity, which allowed us to study how various different transcription dynamics interplay with changes in the 3D genomic organization. Specifically, we analyzed the early activated Tet2 gene; the late activated Sox2 and Nanog genes; the transiently activated Mmp3, Mmp12, CEBP $\alpha$ and Nos1ap genes; the transiently silenced $L$ mo7 genes, the stably silenced Ebf1 gene and the essentially invariant genes Rad23a and Rad23b (Suppl. Figs. 111a and Table 1).

Next, experimental $\mathrm{Hi}-\mathrm{C}$ interaction matrices of the 10 regions at $5 \mathrm{~kb}$ resolution were converted into TADdyn time-dependent spatial restraints (Fig. 1a). Specifically, each $5 \mathrm{~kb}$-bin was represented as a spherical particle of diameter $50 \mathrm{~nm}$, and at each experimental stage the $\mathrm{Hi}-\mathrm{C}$ interaction matrix was converted into harmonic spatial restraints between these particles (Fig. 1b). This conversion follows the simple, yet effective, rationale that pair of particles with a high number of $\mathrm{Hi}-\mathrm{C}$ interactions between them are restrained to stay close in space, while poorly contacting particle pairs are kept far apart $^{8}$ (Methods). The parameters of each imposed restraint between two particles (that is, the spring constant and its equilibrium distance) are then linearly interpolated between the values of the consecutive experimental time points using steered molecular dynamics simulations. This strategy favors the adaptation of the chromatin models to the imposed dynamic restraints (Methods and 
Suppl. Fig. 12). TADdyn restraints are specifically designed to allow smooth structural changes between models of consecutive experimental time-points.

\section{TADdyn simulations are an accurate dynamic representation of the input $\mathrm{Hi}-\mathrm{C}$}

data. To quantify the degree of agreement of the TADdyn 4D models to the timeseries $\mathrm{Hi}-\mathrm{C}$ interaction matrices, contact maps were calculated on an ensemble of 100 models obtained at each time step of the trajectories (Fig. 1d). Next, for each time-point the Spearman correlation coefficient (SCC) of the modeled contact map and the experimental $\mathrm{Hi}-\mathrm{C}$ interaction matrices was computed as a measure of the agreement between the two interactions matrices (Methods). For the 11 loci, the SCC ranged between 0.60 and 0.89 , which is indicative of accurate models ${ }^{35}$ (Fig. 1d for Sox2 simulations and Suppl. Fig. 1-11c for all other loci). Notably, at a fixed cell stage, the Hi-C interaction map correlated best with the contacts maps at the corresponding time of the simulated reprogramming dynamics. These results show that for a diverse set of loci the simulated structures are a reliable 3D representation of two-dimensional $\mathrm{Hi}-\mathrm{C}$ interaction matrices, which effectively reproduce the actual contact pattern at the correct time of the trajectory.

To test whether TADdyn is suited to model processes characterized by gradual chromatin structural transitions, two alternative sets of 100 simulations each for Sox2 were performed by completely deleting the restraints of stages $\mathrm{D} 2$ ( $\triangle \mathrm{D} 2$ simulation) and D6 ( $\triangle \mathrm{D} 6$ simulation). By doubling the time duration between the remaining adjacent transitions ( $\mathrm{B} \alpha$ to D4 and D4 to D8), the total duration of the trajectories was maintained constant. We observed that the missing restraints only marginally affected the results of the simulations. Specifically, the conformations expected to represent the missing cell stages along the trajectories still provided accurate models at the removed stage $\left(\mathrm{SCC}_{\mathrm{D} 2}=0.68\right.$ and $\mathrm{SCC}_{\mathrm{D} 6}=0.75$ in $\triangle \mathrm{D} 2$ and $\Delta \mathrm{D} 6$ simulations, respectively). Additionally, in the case of $\Delta \mathrm{D} 2$, the $\mathrm{Hi}-\mathrm{C}$ interactions map at $\mathrm{D} 2$ correlated best with the contact map computed on the models predicting the D2 stage (for $\triangle \mathrm{D} 6$ the SCC between $\mathrm{Hi}-\mathrm{C}$ at $\mathrm{D} 6$ and models in ES correlated slightly better than the one for $\mathrm{D} 6$; $\mathrm{SCC}_{\mathrm{ES}}=0.76 \mathrm{vs}$. $\left.\mathrm{SCC}_{\mathrm{D} 6}=0.75\right)$. These results suggest that the simulated reprogramming dynamics are dominated by smooth and gradual chromatin rearrangements that can be effectively simulated by TADdyn. 
In the following sections, we present the simulation details the simulations for three representative loci: Sox2 (as a late activated locus), Mmp12 (as a late repressed locus) and Rad23a (as a stably active locus). The detailed results for all the simulated loci are presented in the Suppl. Figs 1-11 and summarized in the last paragraph of the Results section.

\section{Dynamic structural reorganization correlates with local transcriptional}

changes. To explore how the simulated models changed over time, we performed a hierarchical clustering analysis of the correlation matrix between the contact maps of the models and the input Hi-C interaction matrices (Methods, Fig. 2a and Suppl. Figures 1-11d). Hi-C interaction matrices were grouped in well-separated clusters reflecting the expected changes in expression activity of each locus. This clustering indicates that the studied loci changed their topology along with their transcriptional activity. Specifically, for 8 of the 11 simulated loci (that is CEBP $\alpha, E b f 1, L m o 7$, Mmp12, Mmp3, Nos1ap, Sox2, and Tet2) the active and inactive states of the locus were represented by two major clusters. For example, the first cluster of Sox2 comprised the cell stages from B to D4 in which the locus is inactive (RPKM<0.06), while the second cluster includes D6 to PSC when Sox2 is active (RPKM>8.0) (Fig. 2a). Similar observations were made for Mmp12. In contrast, Rad23a showed a less clear partition into two distinct clusters (the 3 first clusters are almost equidistant), likely because the locus remains in an active state during reprogramming with relatively small fluctuations of expression. To further characterize the structural changes associated with changes in transcriptional activity, we performed a clustering analysis of the model structures based on the distance RMSD (dRMSD) values between pairs of 3D models (Methods). As for the matrix-based analysis, the dRMSD clustering reflects the presence of different folding states that correlate with transcriptional activity (Fig. 2b and Suppl. Figures 1-11e).

Gene activation correlates with the appearance of "cage"-like structures. In our simulations (Supplementary videos 1-11), we observed a "caging" effect of the transcription start site (TSS) at the time when a given locus was transcriptionally active. To quantify this observation, we first calculated for each TSS the timedependent changes in its $3 \mathrm{D}$ structural embedding as well its explored volume (Methods, Fig. 3 and Suppl. Figs. 1-11f,g). Notably, TADdyn simulations showed 
that, as cells reprogramed, the TSS for all 11 loci remained largely accessible to other particles during their less transcriptionally active phases (e.g. stages B-D4 for Sox2 and D6-PSC for Mmp12) (Fig. 3a). During the most active stages the TSS particle became embedded in the 3D model (the TSS embedding was between 0.80.95 at the PSC stage for Sox2 and B-D4 for Mmp12) (Fig. 3a). In contrast, the embedding profile of the Rad23a locus was maintained around 0.6 during the entire simulation in parallel with the overall constant transcriptional activity of the gene.

Next, we assessed whether the 3D embedding would also affect the spatial dynamics of the TSS from different loci. For each simulation, we calculated the convex-hull of the TSS particle every 50 time-steps of the trajectory as a proxy of the volume explored by the TSS at each reprogramming stage (Methods and Fig. 3b). The results indicate that the TSS of Sox2 explores a smaller volume in the D6-PSC stages, when the gene is transcriptionally active, compared to the volume explored in the B to D4 stages when the gene is transcriptionally silent. The TSS of Mmp12 acquired increased mobility during reprogramming as its expression levels decreased (compare B-D4 and D6-PSC stages). Interestingly, the explored volume of the Rad23a TSS barely changed during the entire reprograming process. Taken together, these findings, are consistent with previous super-resolution imaging works suggesting that the mobility of the promoter is constrained upon activation ${ }^{36,37}$.

\section{Gene activity correlates with domain borders dynamics and enhancer} proximity. To further characterize the topological transitions between gene expression states, we calculated dynamic changes in TAD insulation score and border strength ${ }^{38}$ using the contact maps of the models along the TADdyn trajectories (Methods). We found that although the number of borders was very constant during the simulation their position often changed. In a striking example involving the Sox2 locus, during the D4-D6 transition there was a clear shift of a border at position chr3:34.60Mb, moving further downstream and converging near the TSS (Fig. 4a). A second border at position chr3:34.74Mb was displaced about $20 \mathrm{~Kb}$ downstream, thereby inducing the topological insulation of a super-enhancer region at chr3:34.70Mb and the TSS (Fig. 4b). These border changes resulted in the formation of a domain of about $120 \mathrm{~kb}$, which included exclusively the Sox 2 gene and its super-enhancer region. The fact that the border strength remained high after gene 
activation at D6 is in agreement with our previously described findings that the Sox2 TSS and its super-enhancer became isolated in a sub-TAD when the gene is transcribed in PSCs (Fig. 4a). Consistent with this observation, the Mmp12 TSS was partially included in a weak domain border at chr9:7.25Mb during transcription. This weak border disappeared during the gene's transition from an active to an inactive state (during the D4-D6 stages), so that the Mmp12 TSS became part of a larger domain at the time of gene silencing. Finally, and with a similar trend, the TSS of the invariantly active gene Rad23a remained part of a domain border, and insulated in an unvaried domain between chr8:84.58-82.86Mb for the entire simulation.

To characterize the elements within the borders of TADs containing transcriptionally active loci, we first identified in the models what we call "active" particles, which had overlapping signals (at least 250bp) of ATAC-seq and H3K4me2 ChIP-seq in any time-point of the reprogramming process ${ }^{34}$ (Methods, Fig. 4b and Suppl. Figures 111g). Upon activation, the Sox 2 gene was positioned spatially close $(<200 \mathrm{~nm})$ to a set of particles containing its super-enhancer (SE) about $120 \mathrm{~kb}$ downstream of the TSS. Notably, and in line with our previous observations ${ }^{34}$, the TSS-SE proximity started at D4, that is before the Sox 2 transcriptional activity could be detected (D6). This spatial proximity was maintained for the rest of the simulation while the Sox2 gene was transcribed. In contrast, the TSS 3D distance profiles of Rad23a and Mmp12 remained overall constant during reprogramming (Fig. 4b) with marginal changes during the simulation.

To further assess to what extend Active particles (Table 2) became proximal to the TSS of the locus, we counted for each reprogramming stage how many of the Active (A) particles became available to the TSS particle, either by spatial proximity only (Active-Proximal particles, AP) or also within the same local domain (ActiveProximal-Domain, APD) (Methods and Fig. 4c). This analysis resulted in a robust trend for all analyzed loci, showing higher numbers of AP and APD particles when the locus is transcriptionally active. Notably, for Sox2 the A, AP and APD particles were increasing together with the locus transcription activity, but the regions containing the annotated super-enhancer were only classified as AP or APD at the D6 stage, that is after the structural changes observed during the simulation at D4 stage. For the Mmp12 simulation the number of A particles did not decrease in the 
inactive stage, yet the numbers of AP or APD decreased consistently with the general trend. The Rad23a locus was instead characterized by a quite constant and high number of $A, A P$ and APD particles consistently with is stable transcription activity during the entire reprogramming process. Altogether, we observed a consistent 3D co-localization of Active and TSS chromatin particles upon activation of the gene, reminiscent of the recently proposed formation of enhancer hubs or condensates $^{39-41}$.

\section{TADdyn simulations indicate that TSS caging is a general structural signature} of transcription activation. To move from anecdotal observations to more general trends, we compared relative expression levels with the dynamic chromatin conformation changes detected during the simulations of all 11 selected loci (Methods and Fig. 5). More specifically, we rescaled RPKM gene expression as well as the other four features obtained from the TADdyn models (that is, the 3D embedding of the TSS particle, the distance to TSS, the volume explored by the TSS, and the number of APD particles) to a 0-to-1 relative scale. Each feature was analyzed for two categories of loci: those that have changes in expression and those that remain active during the entire simulation. The switch category includes the loci that show a substantial change in expression level from high values $(R P K M>1.0)$ to low ones $(\mathrm{RPKM}<0.5)$ or vice versa, and include CEPB $\alpha, M m p 3, M m p 12$, Nos1ap, Ebf1, Sox2, and Nanog. The active simulations were constituted by loci whose activity is always above 1.0 RPKM and included Lmp7, Rad23a, Rad23b, and Tet2. Analysis of these 11 loci revealed that TSS particles tend to be embedded inside the model structure and closer to other active particles when the gene's expression is higher (Fig. 5a, b), exploring less volume (Fig. 5c) and interact with greater numbers of surrounding APD particles (Fig. 5d). These findings suggest that the signatures of the caging effect are common to all 11 loci studied. Additionally, the finding that the numbers of both the AP and APD particles increased with elevated transcription activity (Figs. $\mathbf{4 c}$ and $\mathbf{5 d}$ ) indicates that the formation of a structural cage may have a functional role in promoting and maintaining interactions of the TSS with regulatory regions of the genome.

In summary, our results support what we call here a hit-and-stick model for gene activation. In this model, gene expression is triggered by the formation of a structural 
domain hosting enhancer sequences ( $A D P$ particles), with the TSS being caged inside the newly formed domain. The proximity to the enhancers and the newly established interactions (hit), stabilizes (stick) the spatial position of the locus TSS against the random thermal motion (Fig. 5e). 


\section{DISCUSSION}

Here we have introduced a new computational tool, TADdyn, aimed at studying timedependent dynamics of chromatin domains during a cell fate conversion process that models smooth 3D transitions of chromosome domains. To our knowledge this is the first completely 3C-based data-driven method to simulate structural changes of chromatin over time. TADdyn takes as input a discrete time-series of $\mathrm{Hi}-\mathrm{C}$ interaction maps and converts matrix bins into spherical particles. These are dynamically and spatially restrained using $\mathrm{Hi}-\mathrm{C}$ interactions ${ }^{6}$ and a physics-based chromatin model ${ }^{21}$, effectively integrating restraint-based modeling and polymer physics simulations. For our study, we modeled the dynamic rearrangement of 11 loci during the reprogramming of murine $\mathrm{B}$ cells to pluripotent stem sells ${ }^{34}$, integrating in situ $\mathrm{Hi}-\mathrm{C}$ data obtained for 7 consecutive stages. Molecular dynamics simulations of data at $5 \mathrm{~kb}$ resolution predict viable structural and dynamical transitions between the different cell stages in 3D space. TADdyn is therefore suited to model processes characterized by continuous chromatin structural transitions.

In two alternative simulations of Sox2, we found that missing steps only marginally affect the results of our simulations suggesting that the simulated reprogramming dynamics are dominated by smooth and gradual spatial chromatin rearrangements. In fact, TADdyn has turned out to be useful in determining whether a smooth spatial reorganization of a chromatin region between two consecutive time steps is physically feasible or is hindered by "energetic barriers" between the structures to be simulated.

TADdyn simulations have provided new insights into mechanisms underlying chromatin domain formation and gene expression regulation, extending our current understanding of these processes. First, we found that upon activation, the promoter region establishes interactions with active chromatin particles that contain overlapping signals of chromatin accessibility (ATAC-seq) and regulatory activity (H3K4me2) (Fig. 4). These promoter-active interactions build a local structural domain that embeds the TSS and makes it significantly less accessible to external particles and more spatially constrained against random thermal motion (Figs. 3 and 4). Interestingly, these findings are consistent with previous super-resolution imaging 
studies suggesting that the mobility of the promoter is constrained upon activation $^{36,37}$.

Notably, these active particles with ATAC-seq and H3K4me2 signal often include annotated enhancers as well as newly predicted putative enhancers. Such predicted enhancers can act at long-range, as exemplified by the distal active particles interacting with the Sox 2 promoter. Upon transcription activation, the dynamic formation of local topological domains (cages) inferred here from high-resolution time-resolved $\mathrm{Hi}-\mathrm{C}$ data, is reminiscent of structural or topological motifs (such as rosettes, or cliques) suggested in previous studies using polymer-physics-based models ${ }^{42}$ as well as transcription factories or phase-separated condensates ${ }^{39,40,43,44}$. We speculate that these cages might trigger and maintain the activation of the promoter by maximizing its local association with a series of proximal enhancers. At a larger scale, such single-gene cages might eventually coalescence into larger domains to form large multi-gene condensates.

Based on our findings that the active promoter becomes caged inside a structural domain together with open and active regions we propose a hit-and-stick model for promoter-enhancer transcription regulation (Fig. 5e). Our model predicts that this molecular cage ensures the establishment (hit phase) and the maintenance of a spatial neighborhood where promoter-enhancers interactions are favored for the entire active phase (stick phase). This second phase is accompanied by low accessibility to external particles and stabilization of the TSS against thermal motion. The model also proposes that promoter-enhancer communication ${ }^{45}$ occurs via direct interactions between the distant enhancer and its cognate target gene as previously observed in many studies $33,37,43,44,46-54$. In summary, our hit-and-stick model links the promoter-enhancer direct contact to the formation of local structural domains or "cages".

Our results seem incompatible with alternative mechanisms of gene regulation that do not necessarily depend on promoter-enhancer interactions ${ }^{55,56}$ nor its extension in time ${ }^{57}$. In such an alternative scenario, promoter-enhancer contacts are short-lived but continue to maintain a transcriptionally-competent chromatin environment at promoters to ensure their activity even if long-range interactions are lost. However, as the time difference between consecutive reprogramming samples used to obtain 
$\mathrm{Hi}-\mathrm{C}$ interaction maps was in the order of tens of hours we cannot rule out that at smaller scales cell sub-populations diverge from the general mechanism here proposed. Therefore, this limitation could have precluded detection of other possible dynamic pathways of genome conformation, satisfying only a subset of the input interaction matrices. To address this, TADdyn will have to be implemented in the future using data obtained from finer time-resolved $\mathrm{Hi}-\mathrm{C}$ or imaging-based approaches $^{58,59}$. 


\section{METHODS}

Collection of experimental data. Structural data were obtained from 3C-based chromatin interaction experiments previously generated by $u^{34}$. Specifically, in-situ $\mathrm{Hi}-\mathrm{C}$ datasets were downloaded from the GEO database (accession number GSE96611) for the cells stages from B to PSC cells of the reprogramming process in mouse. The dataset included seven Hi-C experiments: B cells (B), B $\alpha$ cells (after 18h, B $\alpha$ ), day2 (after 48h, D2), day4 (after 48h, D4), day6 (after 48h, D6), day8 (after 48h, D8), and Pluripotent Stem Cells (after about 48h, PSC). The reads were mapped and filtered as previously described ${ }^{34}$. Using these filtered fragments, the genome-wide raw interaction maps were binned at 5 Kilobase $(\mathrm{kb})$ resolution and normalized using "Vanilla" algorithm"60,61 as implemented in TADbit62. Genomic regions were selected around 11 loci of interest (Table 1) (Sox2, Nanog, Mmp3, Mmp12, Ebf1, C/EBP $\alpha$, Rad23a, Rad23b, Lmo7, Nos1ap and Tet2) each characterized by a specific gene expression profile during the cell reprogramming process (Supplementary Figs. 1-11a). The majority of the genes (Sox2, C/EBP $\alpha$, Rad23a, Rad23b, Tet2) span less than $100 \mathrm{~kb}$ and where simulated in the center of 1.5 Megabases (Mb) chromatin regions. For the Nanog locus, the modelled region contained only $1 \mathrm{Mb}$ around the locus after filtering low coverage bins (that is, those with more than $75 \%$ of cells with zero counts), and for the Mmp12 (chr9:7,344,381$7,369,499)$ and Mmp3 (chr9:7,445,822-7,455,975) loci that are contained in the region a single region of $1.5 \mathrm{Mb}$ was considered (chr9:6,650,000-8,150,000). The remaining loci (Ebf1, Lmo7, and Nos1ap) were longer than 100kb and were simulated in 3.0Mb regions centered on the promoter (Fig. 1a and Supplementary Figs. 1-11b). The possibility to study these regions using restraint-based modelling was tested a priori computing the matrix modelling potential (MMP) ${ }^{35}$ on the 70 normalized sub-matrices (that is, the 10 genomic regions and the 7 cell stages). In brief, all Hi-C interaction matrices had MMP scores higher than 0.78 , and contained very few low-coverage bins ( $<0.05 \%$ of the bins), indicating that the restrained-based approach at $5 \mathrm{~kb}$ should provide accurate $3 \mathrm{D}$ models for all the regions ${ }^{35}$ (Supplementary Figs. 1-11b).

Representation of the chromatin region using bead-spring polymer model. Chromatin was represented as a bead-spring polymer describing the effective physical properties of the underlying chromatin ${ }^{18,21}$. Specifically, each $\mathrm{Hi}-\mathrm{C}$ matrix bin of $5 \mathrm{~kb}$ 
was represented as a spherical particle of diameter 50 nanometers $(\mathrm{nm})$ using a compaction ratio of $0.01 \mathrm{bp} / \mathrm{nm}^{63,64}$. Additionally, two non-harmonic potentials were introduced taking into account the excluded volume interaction (purely repulsive Lennard-Jones) and the chain connectivity (Finitely Extensible Nonlinear Elastic, FENE). Both potentials were used as previously described ${ }^{18}$. In the present application, the bending rigidity potential, although it is available in TADdyn, is not used for consistency with the initial models generated using the TADbit software at the B stage (see below). The center of mass of the chromatin chain was tethered to the origin $O=(0.0,0.0,0.0 \mathrm{x}, \mathrm{y}, \mathrm{z}$ coordinates, respectively) of the system using a Harmonic $\left(\mathrm{K}_{\mathrm{t}}=50\right.$., $\left.\mathrm{d}_{\mathrm{eq}}=0.0\right)$, and was simulated inside a cubic box of size $50 \mu \mathrm{m}$ (much larger of the size of the models), which was also centered at $O$.

Encoding of the experimental data into TADdyn restraints. TADdyn accepts one of three possible initial conformations for the chromatin: (i) a polymer chain prepared as a random walk, (ii) an arrangement made of stacked rosettes ${ }^{18}$, or (iii) a previously generated data-driven model. For the 100 time-series simulations performed here, the initial conformations were the 100 optimal models were built using TADbit (https://3DGenomes.org/tadbit) as previously described ${ }^{62}$ for the B cell time point (the first stage of the Hi-C time series). Specifically, we explored all possible combinations of the parameters (lowfreq, upfreq, maxdist, dcutoff) ${ }^{65}$ in the intervals lowfreq=(-3.0,-2.0,-1.0,0.0), upfreq $=(0.0,1 \cdot 0,2 \cdot 0,3.0)$, maxdist $=(150,200,250,300,350,400) \mathrm{nm}$, and dcutoff $=(150,175,200,225,250) \mathrm{nm}$. To select the optimal set of parameters, we computed the correlation of the input $\mathrm{Hi}-\mathrm{C}$ interaction map in the $B$ cell stage and the models contact map at the different distance cutoffs using only the 100 best (that is, with the lowest objective function) models per each triplet of the 500 generated models. Next, per each triplet of parameters, the median Spearman correlation values were computed over the 11 studied loci. The largest median correlation coefficient of 0.78 was obtained for lowfreq=1.0, upfreq=1.0, maxdist $=300 \mathrm{~nm}$, and dcutoff $=225 \mathrm{~nm}$.

Next, the 100 TADbit generated models in B cells were energy minimized using a short run of the Polak-Ribiere version of the conjugate gradient algorithm ${ }^{66}$ to favor smooth adaptations of the implementations of the excluded volume and chain connectivity interaction in TADdyn. 
The optimal TADbit parameters optimized for the B stage (that is, lowfreq of -1.0 , upfreq of 1.0 , and maxdist of $300 \mathrm{~nm}$ ) were then used to define the set distance harmonic restraints of the other time points of the series (Fig. 1b). To adapt the harmonic restraint of a given pair of particles $(i, j)$ between consecutive time points $t_{n}$ and $t_{n+1}$, one of the following 3 possible scenarios was applied (Fig. 1c):

1. If the pair $(i, j)$ was restrained by the same type of distance restraint (Harmonic or LowerBoundHarmonic) in both $t_{n}$ and $t_{n+1}$, the strength $(k)$ and the equilibrium distance $\left(d_{e q}\right)$ of the harmonic were both changed gradually from the values they had in $t_{n}$ to the values they had in $t_{n+1}$.

2. (a) If the distance restraint applied between $(i, j)$ was present at time $t_{n}$, but vanished at time $t_{n+1}$, the strength $k$ was decreased from the value at $t_{n}$ to 0.0 , and the equilibrium distance $d_{e q}$ was kept constant and equal to the value in $t_{n}$.

(b) If the distance restraint was present only at time $t_{n+1}$, the strength $k$ was increased from 0.0 at $t_{n}$ to the value in $t_{n+1}$, and the equilibrium distance $d_{e q}$ was kept constant and equal to the value in $t_{n+1}$.

3. If the pair $(i, j)$ was restrained by different type of distance restraint (Harmonic to LowerBoundHarmonic, or vice versa) in $t_{n}$ and $t_{n+1}$, two distance restraints were defined for $(i, j)$. The restraint which was active at time $t_{n}$ was then switched off as in case $2 \mathrm{a}$, and the one active at time $t_{n+1}$ was switched on as in $2 \mathrm{~b}$.

TADdyn restraint-based dynamics simulations. By applying the previous protocol, the simulation effectively and smoothly modified the underlying restraints during the steered transition from $t_{n}$ to $t_{n+1}$. The dynamics of the system was thus described using the stochastic (Langevin) equation ${ }^{67}$, which was integrated using LAMMPS ${ }^{68}$ (http://lammps.sandia.gov) with values of the particle mass $(m=1.0)$, the friction $\left(\gamma=0.5 T_{L J}{ }^{-1}\right)$, and the integration time step of $d t=0.001 \tau_{L J}$, where $\tau_{L J}$ is the internal time unit ${ }^{69}$. The time-dependent Harmonic and LowerBoundHarmonic restraints were implemented using the Colvars plug-in for LAMMPS ${ }^{70}$ originally introduced for advanced sampling techniques, and here modified to implement the TADdyn transitional restraints. This modified version is freely available at https://github.com/david-castillo/colvars. The transition between consecutive time stages was set to last for $10 \tau_{L J}$, hence a single run to simulate the complete $B$ to PSC reprogramming process passing through the 7 cell stages lasted for $60 \tau$ LJ. In each of the 100 replicates of the reprogramming run, the model conformations were 
stored every $0.1 \tau_{L J}$, and used for further data analysis. To test the predictive power of TADdyn in case of smooth structural rearrangements two additional simulations were performed for the Sox2 locus by removing the restraints of stages D2 ( $\Delta \mathrm{D} 2)$ and D6 ( $\triangle \mathrm{D} 6)$, and by extending the corresponding transitions $(\mathrm{B} \alpha \rightarrow \mathrm{D} 4$ and D4 $\rightarrow$ D8 respectively) to keep constant the total duration of the runs.

\section{Analysis of TADdyn models.}

Time dependent contact maps and TADdyn models assessment. (i) The contact maps were computed at each time step based on the probability within the 100 simulations that pairs of particles are contacting (that is within a distance cut-off of 200nm) (Fig. 2a and Supplementary Figs. 1-11c). (ii) The resulting contact maps along the simulations were clustered. The Spearman's rank correlation coefficients (SCC) was computed with each of the time-series Hi-C interaction map. The SCCs were converted into normalized distances from 0 (max SCC) to 1 ( $\min$ SCC) and used to cluster the contact maps using the Ward hierarchical approach criterion ${ }^{71}$ as implemented in R (Fig. 2b and Supplementary Figs. 1-11d). (iii) For each simulation, the Root Mean Square Deviation (RMSD) between the optimally superimposed models (separated by $1 T_{L J}$ ) was computed. Next, per each model pair, the median dRMSD over the 100 replicates was computed. Finally, the structural clustering analysis was done on the matrix of median dRMSDs by using the Ward hierarchical approach criterion as implemented in R (Fig. 2c and Supplementary Figs. 1-11e).

Time dependent measures of the TADdyn structures. (i) The accessibility (A) of each particle in the ensemble of models was calculated using TADbit with parameters nump $=100$, radius $=50 \mathrm{~nm}$, and super-radius $=200 \mathrm{~nm}$. The accessibility (A) transformed into embedding $(E=1.0-A)$ that is a measure of the propensity of a particle to be caged inside an internal cavity. The embedding ranges from 0.0 meaning that the particle is located on the interface of the model to 1.0 when the particle is closely surrounded by other particles inside the model (caged) (Fig. 3a and Supplementary Figs. 1-11f). (ii) The explored volume per particle every $5 \tau_{L J}$ of trajectory was calculated. Specifically, all trajectories were partitioned in time intervals of $5 \tau_{L J}$. In each time interval, the 50 positions (i.e. one every $0.1 \tau_{L J}$ ) occupied by the particle $i$ were considered, and the convex-hull embedding these 50 
positions was calculated. In a given time interval, the average (over the 100 replicate runs) convex-hull volume explored by particle $i$ was considered as the typical volume explored by the model particle $i$ during the time interval (Fig. 3b and Supplementary Fig.s 1-11g). (iii) The spatial distance between selected pairs of particles was computed for the ensemble of simulations as the Euclidian distance in nanometers using TADbit (Fig. 4a and Supplementary Figs. 1-11h).

Time dependent insulation score analysis of TADdyn models. To study the partitioning of the models into structural domains (reminiscent of TADs ${ }^{72-74}$ ), the insulation score (I-score) analysis ${ }^{38}$ was performed on the models contact maps using the command line --is 100000 --ids 50000 --ez --im mean --nt 0.1 --bmoe 3. The called domain borders, whose border strength was deemed significant by the Iscore pipeline, were used for further analysis (Fig. 4b and Supplementary Fig.s 111i).

Active particles analysis. In each cell stage, we classified some model particles (5kb) into one of 3 possible categories (Supplementary Table 2, Fig. 4c and Supplementary Figs. 1-11I and $\mathbf{m}$ ): Active $(A)$ are particles hosting at least one overlapping 250bp-peak of ATAC-seq and H3K4me2 (ATAC-seq and H3K4me2 data were obtained from our previous work ${ }^{34}$ ), Active-Proximal (AP) are Active particles that are close to the TSS particle of the gene either in absolute terms (spatial distance $<200 \mathrm{~nm}$ ) or in relative terms (spatial distance < half of average distance at the genomic separation) for at least half of the duration of the stage. Active-ProximalDomain (ADP) are AP particles that are inside the local domain containing the TSS (see insulation score analysis above).

The statistical comparison between the distributions of the different quantities analyzed (spatial distance, embedding, and explored volume) was performed by bootstrapping (re-sampling with replacement). The re-sampling procedure is repeated 1,000 times for a fixed number of elements, specifically 200 for the comparisons in Fig. 4c and 1000 for the ones in Fig. 5a-c). For the 1,000 comparisons of the re-sampled distributions, we tested for significant differences a Wilcoxon test using $\mathrm{R}$. The higher $\mathrm{p}$-value was assigned to the corresponding pair of distributions under comparison. The comparisons that resulted in maximum $p$ values $<0.01$ were deemed to be significantly different. 


\section{DATA AVAILABILITY}

The TADdyn approach is currently available as part of the TADbit Github repository (https://github.com/david-castillo/TADbit/tree/poly). The modified version of the COLVAR plug-in for LAMMPS is available at the Github repository (https://github.com/david-castillo/colvars)

\section{ACKNOWLEDGEMENTS}

We thank all the current and past members of the Marti-Renom lab for their continuous discussions and support to the development of TADdyn.

\section{FUNDING}

This work was partially supported by the European Research Council under the $7^{\text {th }}$ Framework Program FP7/2007-2013 (ERC grant agreement 609989 to M.A.M-R. and T.G.), the European Union's Horizon 2020 research and innovation programme (grant agreement 676556 to M.A.M-R.) and the Spanish Ministerio de Ciencia, Innovación y Universidades (BFU2013-47736-P and BFU2017-85926-P to M.A.M-R. as well as IJCI-2015-23352 to I.F.). R.S. is supported by the Netherlands Organization for Scientific Research (VENI 91617114) and an Erasmus MC Fellowship. We also knowledge support from 'Centro de Excelencia Severo Ochoa 2013-2017', SEV-2012-0208 and the CERCA Programme/Generalitat de Catalunya to the CRG.

\section{CONFLICT OF INTEREST}

None declared. 


\section{REFERENCES}

1. de Laat, W. \& Duboule, D. Topology of mammalian developmental enhancers and their regulatory landscapes. Nature 502, 499-506 (2013).

2. Dekker, J. \& Mirny, L. The 3D Genome as Moderator of Chromosomal Communication. Cell 164, 1110-21 (2016).

3. Stadhouders, R., Filion, G.J. \& Graf, T. Transcription factors and 3D genome conformation in cell-fate decisions. Nature 569, 345-354 (2019).

4. Spielmann, M., Lupianez, D.G. \& Mundlos, S. Structural variation in the 3D genome. Nat Rev Genet 19, 453-467 (2018).

5. Dekker, J., Rippe, K., Dekker, M. \& Kleckner, N. Capturing chromosome conformation. Science 295, 1306-11 (2002).

6. Serra, F. et al. Restraint-based three-dimensional modeling of genomes and genomic domains. FEBS Lett 589, 2987-95 (2015).

7. Jhunjhunwala, S. et al. The 3D structure of the immunoglobulin heavy-chain locus: implications for long-range genomic interactions. Cell 133, 265-79 (2008).

8. Baù, D. et al. The three-dimensional folding of the alpha-globin gene domain reveals formation of chromatin globules. Nat Struct Mol Biol 18, 107-14 (2011).

9. Umbarger, M.A. et al. The three-dimensional architecture of a bacterial genome and its alteration by genetic perturbation. Mol Cell 44, 252-64 (2011).

10. Tjong, $\mathrm{H}$. et al. Population-based 3D genome structure analysis reveals driving forces in spatial genome organization. Proc Natl Acad Sci U S A 113, E1663-72 (2016).

11. Trussart, M. et al. Defined chromosome structure in the genome-reduced bacterium Mycoplasma pneumoniae. Nat Commun 8, 14665 (2017).

12. Paulsen, J. et al. Chrom3D: three-dimensional genome modeling from $\mathrm{Hi}-\mathrm{C}$ and nuclear lamin-genome contacts. Genome Biol 18, 21 (2017).

13. Dekker, J., Marti-Renom, M.A. \& Mirny, L.A. Exploring the three-dimensional organization of genomes: interpreting chromatin interaction data. Nat Rev Genet 14, 390-403 (2013).

14. Junier, I., Spill, Y.G., Marti-Renom, M.A., Beato, M. \& le Dily, F. On the demultiplexing of chromosome capture conformation data. FEBS Lett 589, 3005-13 (2015).

15. Barbieri, M. et al. Complexity of chromatin folding is captured by the strings and binders switch model. Proc Natl Acad Sci U S A 109, 16173-8 (2012).

16. Giorgetti, L. et al. Predictive polymer modeling reveals coupled fluctuations in chromosome conformation and transcription. Cell 157, 950-63 (2014).

17. Tiana, G. \& Giorgetti, L. Integrating experiment, theory and simulation to determine the structure and dynamics of mammalian chromosomes. Curr Opin Struct Biol 49, 11-17 (2018).

18. Rosa, A. \& Everaers, R. Structure and dynamics of interphase chromosomes. PLoS Comput Biol 4, e1000153 (2008).

19. Jost, D., Carrivain, P., Cavalli, G. \& Vaillant, C. Modeling epigenome folding: formation and dynamics of topologically associated chromatin domains. Nucleic Acids Res 42, 9553-61 (2014).

20. Brackley, C.A., Johnson, J., Kelly, S., Cook, P.R. \& Marenduzzo, D. Simulated binding of transcription factors to active and inactive regions folds human chromosomes into loops, rosettes and topological domains. Nucleic Acids Res 44, 3503-12 (2016).

21. Di Stefano, M., Paulsen, J., Lien, T.G., Hovig, E. \& Micheletti, C. Hi-C-constrained physical models of human chromosomes recover functionally-related properties of genome organization. Sci Rep 6, 35985 (2016).

22. Fudenberg, G. et al. Formation of Chromosomal Domains by Loop Extrusion. Cell Rep 15, 2038-49 (2016).

23. Tiana, G. et al. Structural Fluctuations of the Chromatin Fiber within Topologically Associating Domains. Biophys J 110, 1234-45 (2016). 
24. Fudenberg, G. \& Imakaev, M. FISH-ing for captured contacts: towards reconciling FISH and 3C. Nat Methods 14, 673-678 (2017).

25. Sanborn, A.L. et al. Chromatin extrusion explains key features of loop and domain formation in wild-type and engineered genomes. Proc Natl Acad Sci U S A 112, E6456-65 (2015).

26. Brackley, C.A. et al. Extrusion without a motor: a new take on the loop extrusion model of genome organization. Nucleus 9, 95-103 (2018).

27. Naumova, N. et al. Organization of the mitotic chromosome. Science 342, 948-53 (2013).

28. Gibcus, J.H. et al. A pathway for mitotic chromosome formation. Science 359(2018).

29. Wang, Y. et al. Reprogramming of Meiotic Chromatin Architecture during Spermatogenesis. Mol Cell 73, 547-561 e6 (2019).

30. Alavattam, K.G. et al. Attenuated chromatin compartmentalization in meiosis and its maturation in sperm development. Nat Struct Mol Biol 26, 175-184 (2019).

31. Patel, L. et al. Dynamic reorganization of the genome shapes the recombination landscape in meiotic prophase. Nat Struct Mol Biol 26, 164-174 (2019).

32. Le Dily, F. et al. Distinct structural transitions of chromatin topological domains correlate with coordinated hormone-induced gene regulation. Genes Dev 28, 2151-62 (2014).

33. Bonev, B. et al. Multiscale 3D Genome Rewiring during Mouse Neural Development. Cell 171, 557-572 e24 (2017).

34. Stadhouders, R. et al. Transcription factors orchestrate dynamic interplay between genome topology and gene regulation during cell reprogramming. Nat Genet 50, 238-249 (2018).

35. Trussart, M. et al. Assessing the limits of restraint-based 3D modeling of genomes and genomic domains. Nucleic Acids Res 43, 3465-77 (2015).

36. Germier, T. et al. Real-Time Imaging of a Single Gene Reveals Transcription-Initiated Local Confinement. Biophys J 113, 1383-1394 (2017).

37. Chen, H. et al. Dynamic interplay between enhancer-promoter topology and gene activity. Nat Genet 50, 1296-1303 (2018).

38. Crane, E. et al. Condensin-driven remodelling of $X$ chromosome topology during dosage compensation. Nature 523, 240-4 (2015).

39. Sabari, B.R. et al. Coactivator condensation at super-enhancers links phase separation and gene control. Science 361(2018).

40. Cho, W.K. et al. Mediator and RNA polymerase II clusters associate in transcriptiondependent condensates. Science 361, 412-415 (2018).

41. Miguel-Escalada, I. et al. Human pancreatic islet 3D chromatin architecture provides insights into the genetics of type 2 diabetes. bioRxiv, 400291 (2018).

42. Junier, I., Martin, O. \& Kepes, F. Spatial and topological organization of DNA chains induced by gene co-localization. PLoS Comput Biol 6, e1000678 (2010).

43. Carter, D., Chakalova, L., Osborne, C.S., Dai, Y.F. \& Fraser, P. Long-range chromatin regulatory interactions in vivo. Nat Genet 32, 623-6 (2002).

44. Kagey, M.H. et al. Mediator and cohesin connect gene expression and chromatin architecture. Nature 467, 430-5 (2010).

45. Schoenfelder, S. \& Fraser, P. Long-range enhancer-promoter contacts in gene expression control. Nat Rev Genet (2019).

46. Mitchell, J.A. \& Fraser, P. Transcription factories are nuclear subcompartments that remain in the absence of transcription. Genes Dev 22, 20-5 (2008).

47. Papantonis, A. et al. Active RNA polymerases: mobile or immobile molecular machines? PLoS Biol 8, e1000419 (2010).

48. Deng, W. et al. Controlling long-range genomic interactions at a native locus by targeted tethering of a looping factor. Cell 149, 1233-44 (2012).

49. Andrey, G. et al. A switch between topological domains underlies HoxD genes collinearity in mouse limbs. Science 340, 1234167 (2013). 
50. Lee, K., Hsiung, C.C., Huang, P., Raj, A. \& Blobel, G.A. Dynamic enhancer-gene body contacts during transcription elongation. Genes Dev 29, 1992-7 (2015).

51. Mifsud, B. et al. Mapping long-range promoter contacts in human cells with high-resolution capture Hi-C. Nat Genet 47, 598-606 (2015).

52. Spitz, F. Gene regulation at a distance: From remote enhancers to 3D regulatory ensembles. Semin Cell Dev Biol 57, 57-67 (2016).

53. Morgan, S.L. et al. Manipulation of nuclear architecture through CRISPR-mediated chromosomal looping. Nat Commun 8, 15993 (2017).

54. Rege, M. et al. LADL: Light-activated dynamic looping for endogenous gene expression control. bioRxiv, 349340 (2018).

55. Gupta, R.M. et al. A Genetic Variant Associated with Five Vascular Diseases Is a Distal Regulator of Endothelin-1 Gene Expression. Cell 170, 522-533 e15 (2017).

56. Benabdallah, N.S. et al. PARP mediated chromatin unfolding is coupled to long-range enhancer activation. bioRxiv, 155325 (2017).

57. Alexander, J.M., Guan, J., Huang, B., Lomvardas, S. \& Weiner, O.D. Live-Cell Imaging Reveals Enhancer-dependent Sox2 Transcription in the Absence of Enhancer Proximity. bioRxiv, 409672 (2018).

58. Dekker, J. Mapping the 3D genome: Aiming for consilience. Nat Rev Mol Cell Biol 17, 741-742 (2016).

59. Nir, G. et al. Walking along chromosomes with super-resolution imaging, contact maps, and integrative modeling. PLoS Genet 14, e1007872 (2018).

60. Imakaev, M. et al. Iterative correction of $\mathrm{Hi}-\mathrm{C}$ data reveals hallmarks of chromosome organization. Nat Methods 9, 999-1003 (2012).

61. Rao, S.S. et al. A 3D map of the human genome at kilobase resolution reveals principles of chromatin looping. Cell 159, 1665-80 (2014).

62. Serra, F. et al. Automatic analysis and 3D-modelling of Hi-C data using TADbit reveals structural features of the fly chromatin colors. PLoS Comput Biol 13, e1005665 (2017).

63. Harp, J.M., Hanson, B.L., Timm, D.E. \& Bunick, G.J. Asymmetries in the nucleosome core particle at 2.5 A resolution. Acta Crystallogr D Biol Crystallogr 56, 1513-34 (2000).

64. Finch, J.T. \& Klug, A. Solenoidal model for superstructure in chromatin. Proc Natl Acad Sci U SA 73, 1897-901 (1976).

65. Bau, D. \& Marti-Renom, M.A. Structure determination of genomic domains by satisfaction of spatial restraints. Chromosome Res 19, 25-35 (2011).

66. Polak, E. \& Ribière, G. Note sur la convergence de directions conjugées. Rev Fran Informat Recherche Op 16, 35-43 (1969).

67. Schneider, T. \& Stoll, E. Molecular-dynamics study of a three-dimensional one-component model for distortive phase transitions. Phys. Rev. B 17, 1302 (1978).

68. Plimpton, S. Fast Parallel Algorithms for Short-Range Molecular Dynamics. J Comp Phys 117, 1-19 (1995).

69. Kremer, K. \& Grest, G.S. Dynamics of entangled linear polymer melts: A molecular-dynamics simulation. .J. Chem. Phys. 92, 5057-5086 (1990).

70. Fiorin, G., Klein, M.L. \& Hénin, J. Using collective variables to drive molecular dynamics simulations. Molecular Physics 111, 3345-3362 (2013).

71. Szekely, G. \& Rizzo, M. Hierarchical Clustering via Joint Between-Within Distances: Extending Ward's Minimum Variance Method. Journal of Classification 22, 151 (2005).

72. Sexton, T. et al. Three-dimensional folding and functional organization principles of the Drosophila genome. Cell 148, 458-72 (2012).

73. Nora, E.P. et al. Spatial partitioning of the regulatory landscape of the X-inactivation centre. Nature 485, 381-5 (2012).

74. Dixon, J.R. et al. Topological domains in mammalian genomes identified by analysis of chromatin interactions. Nature 485, 376-80 (2012). 
bioRxiv preprint doi: https://doi.org/10.1101/642009; this version posted May 20, 2019. The copyright holder for this preprint (which was

not certified by peer review) is the author/funder, who has granted bioRxiv a license to display the preprint in perpetuity. It is made available under aCC-BY 4.0 International license. 


\section{FIGURES}

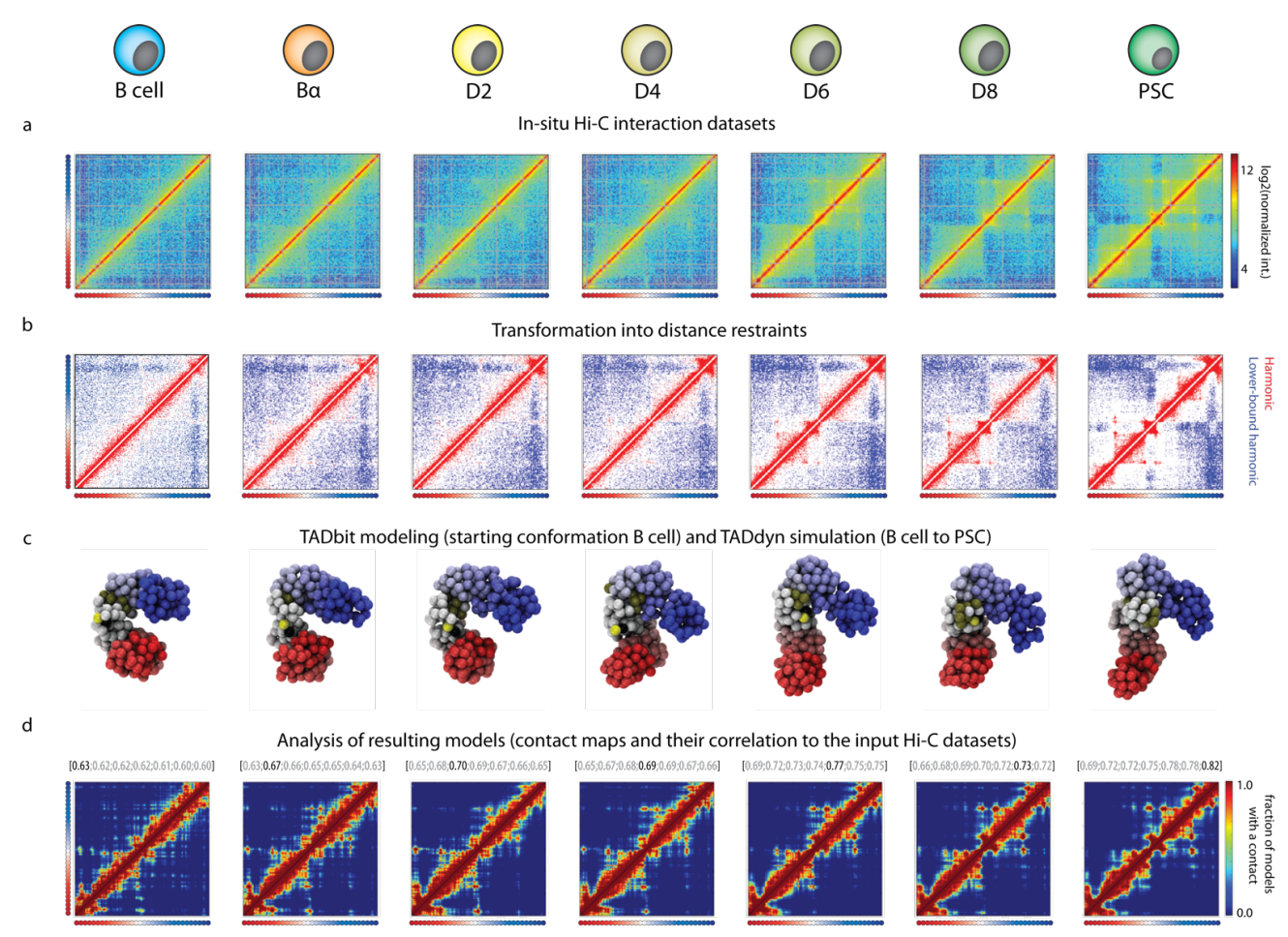

Figure 1. The TADdyn key steps for simulating 4D dynamic changes in a locus.

The shown example corresponds to the reprogramming dynamics for the Sox2 locus from B cells to PSC. a Data collection. In-situ normalized Hi-C interaction matrices ${ }^{34}$ for the region of $1.5 \mathrm{Mb}$ centered around the Sox 2 promoter. $\mathbf{b}$ Distance restraints definition. Both LowerBound- (blue) and Harmonic (red) spatial restraints are obtained by filtering the $\mathrm{Hi}-\mathrm{C}$ interaction maps using the optimal triplet of TADbit parameters. c TADdyn steered dynamics runs. The loci are represented as polymers made of spherical particles containing $5 \mathrm{~kb}$ each a bin of the input $\mathrm{Hi}-\mathrm{C}$ matrix. Particles are colored from red (start of the modeled region) to blue (end of the modeled region). The TSS of the locus of interest is represented by a black particle. The models for all the stages (that is, from B to PSC) were dynamically built by TADdyn. Only the models at the experimental time-points are shown, but TADdyn allows to visualize the entire dynamics filling the blanks between stages (Suppl. Videos 1-11). d Contact maps (<200 nm distance cutoff) of models during the simulation were used to assess their accuracy by means of its correlation with the $\mathrm{Hi}-\mathrm{C}$ input matrices. The Spearman correlation coefficients (SCCs) of the contact maps with the seven $\mathrm{Hi}-\mathrm{C}$ input matrices are shown with the coefficient in black letters corresponding to the time point of the column. 


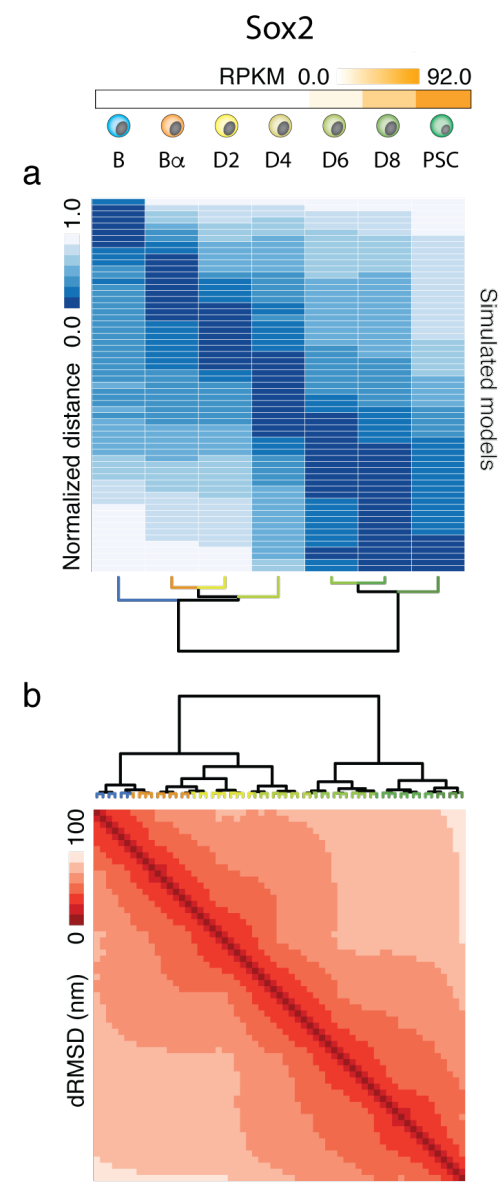

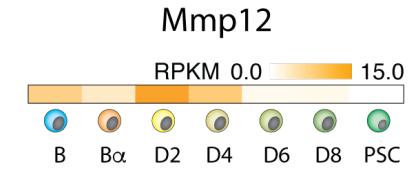
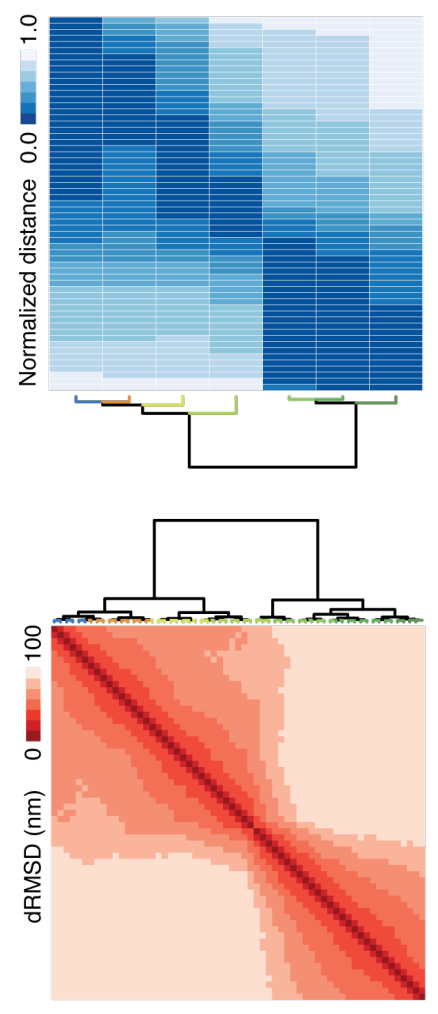

$\operatorname{Rad} 23 a$
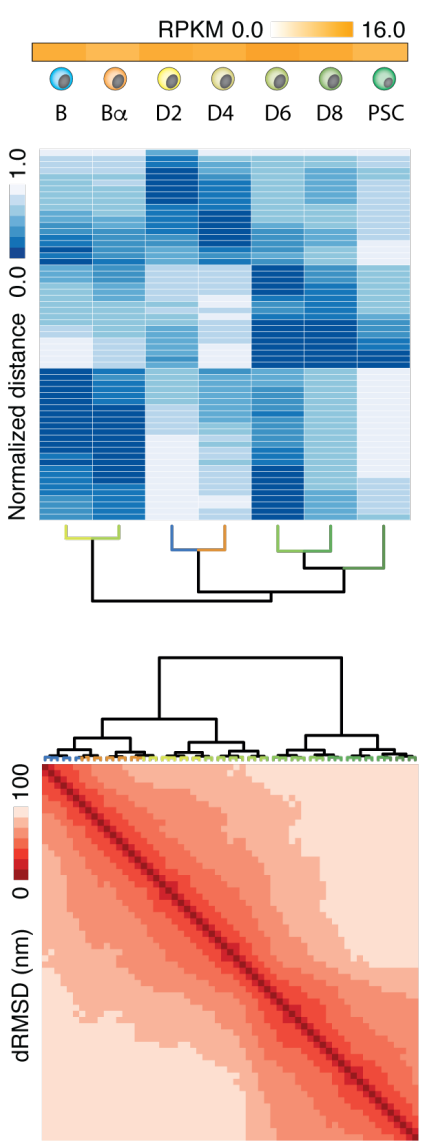

Figure 2. Dynamic structural chromatin reorganization linked to transcriptional activity. Top of the figure indicates expression level (RPKM) for each of the selected loci at each reprogramming stage ${ }^{34}$. a. Normalized spearman rank correlation profiles computed for each pair of 60 model contact maps, that is one map every 1 time step of the simulation, against each of the seven Hi-C interaction maps obtained during the reprogramming process. b. Model $3 D$ structural clustering based on distance RMSD (dRMSD) between all models within a simulation. 
Sox2

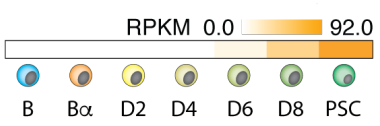

a

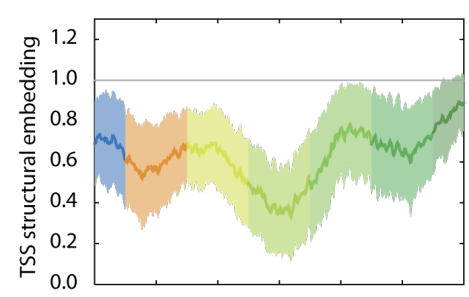

b

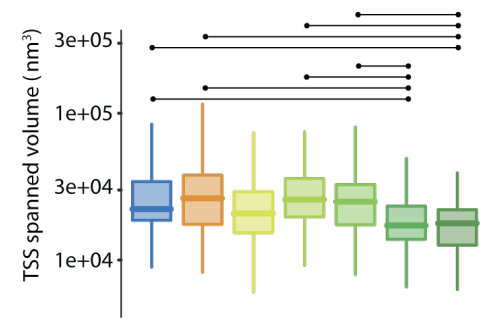

Mmp12
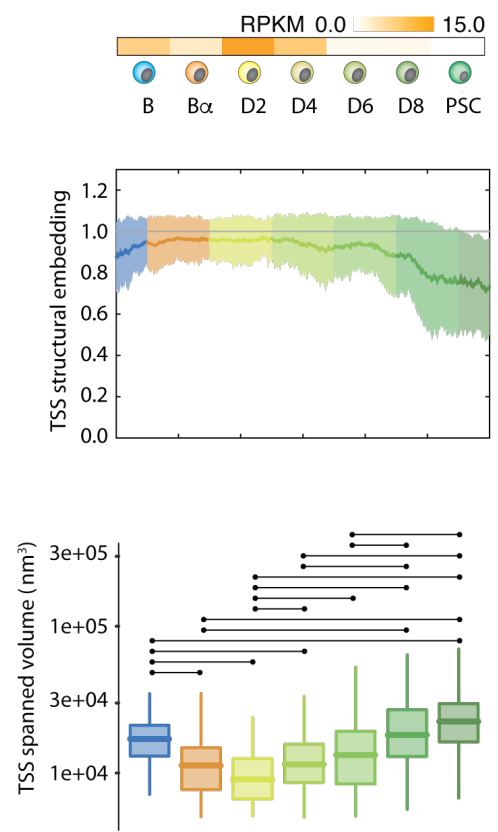

$\operatorname{Rad} 23 a$
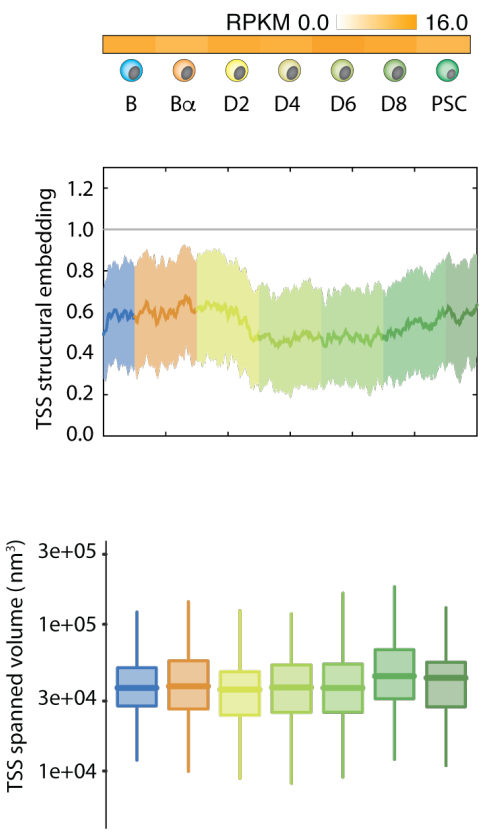

Figure 3. Gene activity correlates with TSS structural embedding and spanned volume. Top of the figure indicates expression level (RPKM) for each of the selected loci at each reprogramming stage ${ }^{34}$. a Dynamic structural embedding of the TSS particle. b Volume explored by the TSS particle during the various stages of the reprogramming dynamics. Connecting lines between distributions indicate they are statistically different ( $p<0.01$, Wilcoxon rank sum test, Methods). 

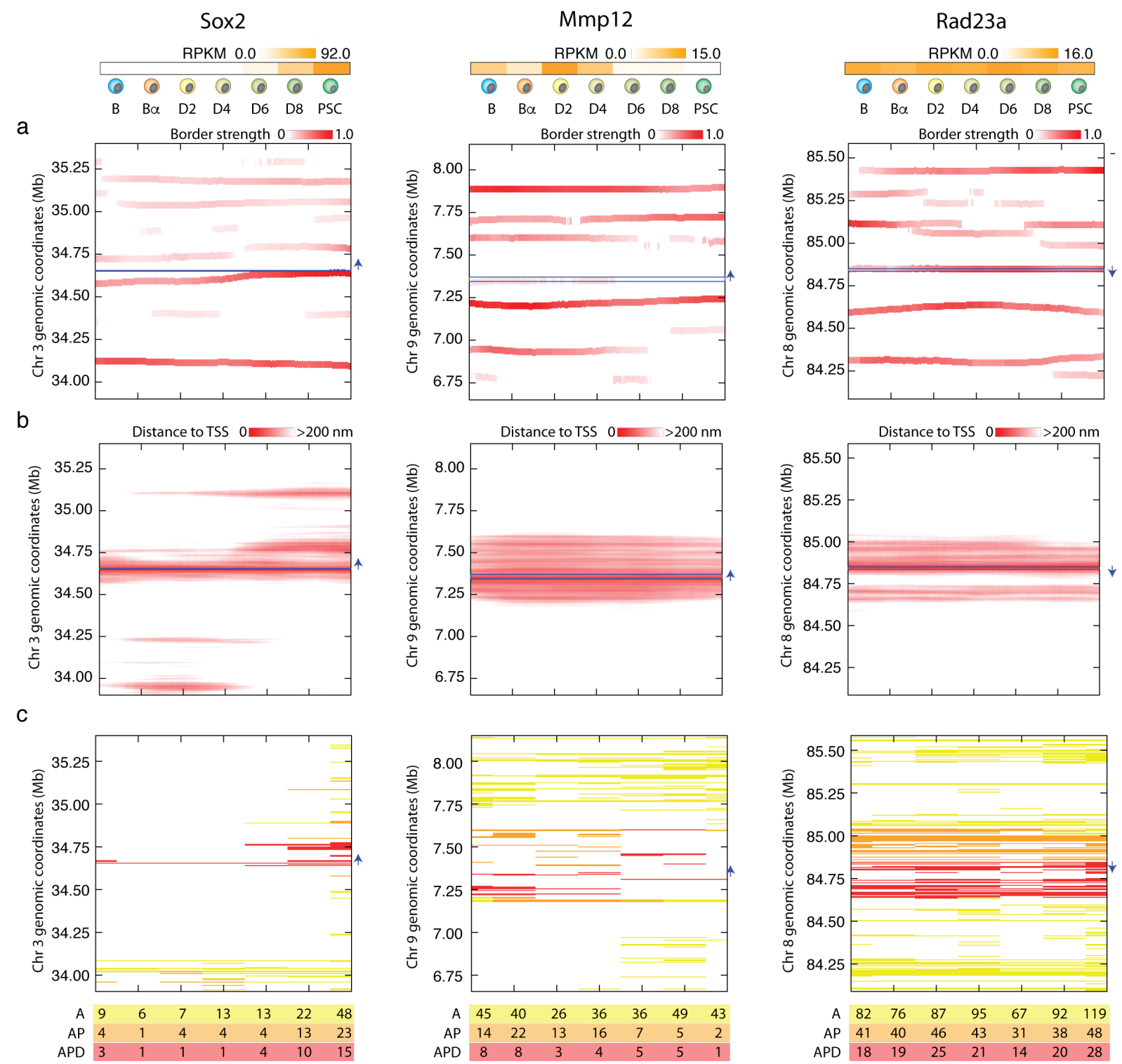

Figure 4. Gene activity correlates with domain borders and enhancer proximity. Top of the figure indicates expression level (RPKM) for each of the selected loci at each reprogramming stage ${ }^{34}$. a Time dependent position of the domain borders as defined by the insulation score analysis on the contact maps derived from the models. The position of the loci in the graph is indicated by a blue horizontal line and its transcriptional orientation is indicated by a blue arrow. $\mathbf{b}$ Heat maps of the distances between the TSS particle and all the other model particles as a function of time. c Active particles classification into proximal to the TSS (AP, yellow), within the domain ( $A D$, orange), and within the domain and proximal to the TSS (APD, red). The bottom table shows the number of particles in each category for each reprogramming stage. 

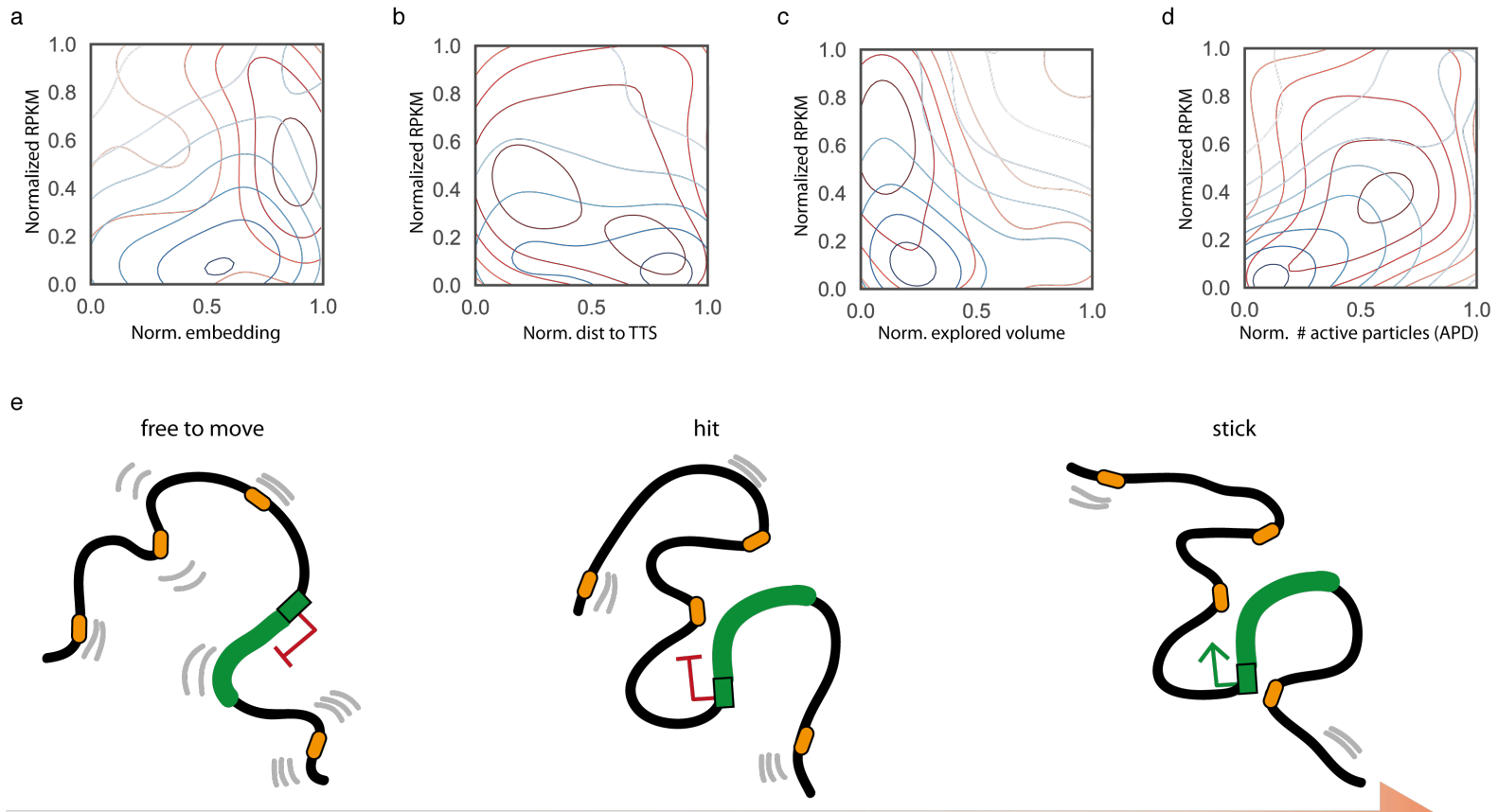

Time and expression levels

Figure 5. The hit-and-stick model of dynamic gene activation. Density distributions of normalized RPKM expression levels for two sets of simulated loci (that is, continuously active loci in red and loci that switch activity state during simulation in blue) with respect to: a. normalized TSS embedding, b. normalized distance to TSS, c. normalized explored volume, and $\mathbf{d}$. normalized number of active APD particles. e. Cartoon of the Hit-and-Stick model for gene activation suggested by the TADdyn simulations over 11 loci. 